\title{
SC-FDE Based MIMO Uplink Transmission over Infrared Communication Channels
}

\author{
Omer Narmanlioglu ${ }^{1}$, Bugra Turan ${ }^{2}$, Refik Caglar Kizilirmak ${ }^{3}$, Sinem Coleri Ergen ${ }^{2}$, and Murat Uysal ${ }^{1}$ \\ ${ }^{1}$ Department of Electrical and Electronics Engineering, Ozyegin University, Istanbul, Turkey \\ E-mail: omer.narmanlioglu@ozu.edu.tr, murat.uysal@ozyegin.edu.tr \\ ${ }^{2}$ Department of Electrical and Electronics Engineering, Koc University, Istanbul, Turkey \\ E-mail: bturan14@ku.edu.tr, sergen@ku.edu.tr \\ ${ }^{3}$ Department of Electrical and Electronics Engineering, Nazarbayev University, Astana, Kazakhstan \\ E-mail: refik.kizilirmak@nu.edu.kz
}

\begin{abstract}
In this paper, we propose a multiple-input multipleoutput (MIMO) uplink transmission scheme for optical wireless communication applications. The transmission is based on optical single-carrier frequency domain equalization due to its low complexity where the signal is transmitted over infrared communication channels. Based on non-sequential ray tracing, we first obtained realistic infrared MIMO channel impulse responses including low-pass filter effect of infrared light-emitting-diodes. We then investigate the performance of bit-error-rate and peak to average power ratio with respect to different modulation orders under the consideration of spatial multiplexing as MIMO mode.

Index Terms-MIMO, Frequency Domain Equalizer, Visible Light Communication.
\end{abstract}

\section{INTRODUCTION}

Visible light communication (VLC) using light emitting diodes (LEDs) as transmitter and photodetectors (PDs) as receiver is an emerging communication technology for an alternative short range optical wireless communications. It enables low-cost, low-complexity, energy-efficient and secure solutions for various applications. One application area of the VLC is indoor wireless communications, as it enables radio frequency (RF) interference free and reliable high data-rate communication networks inside buildings.

However, VLC inherits certain limitations to provide high speed communications such as limited modulation bandwidth and non-linear characteristics of optical transmitters, narrow receiver field of view (FoV) and the multipath dispersion sourced from reflections. Considering commercial white LEDs, white phosphor coating on top of blue LED chip limits the modulation bandwidth of the LED. On the other hand, receiver FoV is restricted to limit noise from other light sources. Furthermore, multi-path dispersion sourced from object reflections poses a serious challenge resulting with intersymbol interference (ISI) at the receiver degrading system performance.

To date, certain challenges are addressed with the realization of indoor VLC networks. In order to overcome the limited

The work of M. Uysal was supported by the Turkish Scientific and Research Council (TUBITAK) under Grant 215E311. Sinem Coleri Ergen acknowledges the financial support by the Turkish Academy of Sciences (TUBA) within the Young Scientist Award Program (GEBIP) and METU-Prof. Dr. Mustafa Parlar Foundation Research Encouragement Award. modulation bandwidth of LEDs and multi-path dispersion, orthogonal frequency-division multiplexing (OFDM), providing reliable high data-rate wireless communications over time domain dispersive channel, has been adopted into VLC. Nonnegative and real-valued signal requirements and non-coherent characteristics of LEDs are considered with various optical OFDM schemes such as direct current biased optical OFDM (DCO-OFDM) [1], asymmetrically-clipped optical OFDM (ACO-OFDM) [2], flip-OFDM [3] (or unipolar OFDM (UOFDM) [4]) and its enhanced version, enhanced U-OFDM (eU-OFDM) [5]. Optical OFDM is known to handle ISI with the division of wide-band channel into narrow-band subchannels and cyclic prefix (CP) insertion longer than the delay spread of the channel. However, signal clipping due to limited linear working region of LEDs and high peak to average power ratio (PAPR) characteristics of OFDM signals necessitated alternative spectrally efficient modulation schemes for VLC. Thereby, optical carrier-less amplitude and phase (CAP) and pulse amplitude modulation (PAM) have been investigated as promising alternatives of OFDM due to their lower PAPR requirements [6].

Single carrier frequency domain equalization (SC-FDE) has been shown to be an appealing equalization scheme for very long impulse memory broadband wireless channels [7]. Channel distortions are compensated at the receiver for SCFDE scheme. Thus, optical transmitter such as LED, laser diode and amplifier non linearities do not play a vital role as it is the case with OFDM. As OFDM signal envelope is characterized by a wide dynamic range for high order discrete Fourier transform (DFT), transmitter front end requirements increase when OFDM usage is considered. In the literature, [8], [9] compared various SC-FDE methods with discrete multi-tone modulation (DMT) and demonstrated performance enhancements due to reduced PAPR. However, with the increasing constellation size of OFDM signals, PAPR advantage of single carrier systems with respect to OFDM signals decreases [10]. On the other hand CAP is proposed as an alternative to OFDM with low PAPR requirements. However time domain equalization complexity and high rate resolution analog to digital converter (AD) requirements are considered to be limitations for CAP practical implementation. 


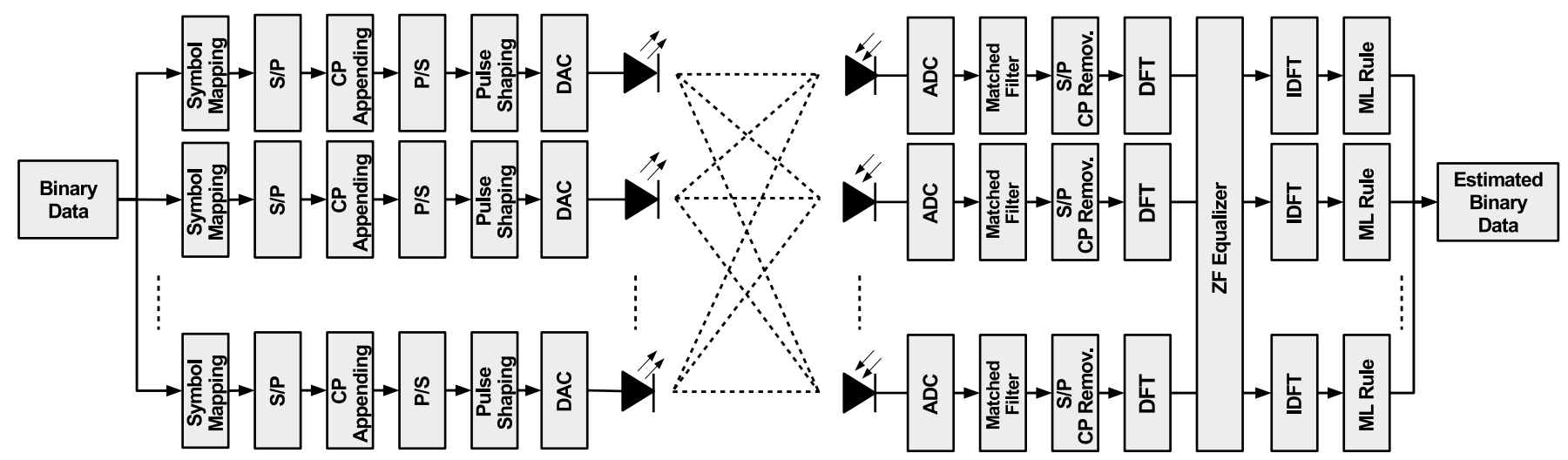

Figure 1: MIMO SC-FDE based transmission.

In indoor VLC, multiple LEDs in a single room makes downlink multiple input multiple output (MIMO) a promising candidate to provide continuous communication with high data rates. Hence, MIMO -OFDM for downlink indoor VLC is studied in [11]-[13]. On the other hand, authors in [14] studied indoor MIMO VLC with SC-FDE, experimentally up to 85 $\mathrm{cm}$ distance under the consideration of direct line-of-sight (LoS) link. However, reflections due to walls, floor and objects such as desk, chair should be addressed to fully evaluate the proposed VLC scheme performance.

In addition to downlink VLC, uplink VLC has recently gained attention to provide high speed transmissions in RF denied environments and sensitive places including security applications. However, visible light LED usage is not feasible as the illumination can be uncomfortable to human eyes. Thus, infrared (IR) LED utilization is promising to fulfill practical uplink VLC requirements. Very few studies considered IR LED usage for uplink optical communications such as authors in [15] investigated IR usage for uplink VLC with the proposed adaptive beam steering IR system. However, none of the studies to date, considered practical VLC uplink channel with the usage of IR LED and SC-FDE-MIMO. To fill this gap, in this study, we evaluate SC-FDE based MIMO uplink transmission scheme considering the practical indoor IR channel. The contributions of this study are twofold. First, we investigate the VLC uplink channel with IR LED utilizing ray tracing capability of Zemax software. Then, we evaluated the biterror-rate (BER) and PAPR performance of SC-FDE MIMO transmission with respect to different modulation orders.

The remainder of the paper is organized as follows. In Section II, we describe SC-FDE based MIMO transmission model. In Section III, we present uplink IR channel model generated by ray-tracing simulations. In Section IV, BER and PAPR results obtained through Monte Carlo simulations are depicted. We finally conclude the work in Section V.

Notation: $\|.\|^{2}$ and $[.]^{\mathrm{T}}$ denote Euclidean distance and transpose respectively. $\otimes$ is convolution operator. $F\{$.$\} represents$ the continuous Fourier transform. $Q($.$) is the tail probability of$ standard normal distribution. $\mathbf{E}[$.$] is the statistical expectation.$

\section{System MODEL}

Our uplink transmission model is based on optical SC-FDE. In optical SC-FDE, the binary information is first mapped to real non-negative symbols $\left[\begin{array}{llllll}s_{0} & s_{1} & s_{2} & \ldots & s_{N-1}\end{array}\right]$ based on the deployed constellation scheme such as unipolar PAM [16] where $N$ is frame size. Intensity levels of unipolar $M-$ PAM are given by

$$
I_{c}=c \frac{2 I}{M-1} \quad c \in\{0,1 \ldots M-1\}
$$

where $I$ is the mean optical power. In order to mitigate the effect of the multi-path channel response, $\mathrm{CP}$ with a length of $N_{C P}$ is added to the beginning of each frame, then parallelto-serial conversion is performed over parallel frames. $N_{C P}$ is selected to be longer than the multipath channel length. Thus, knowing channel delay plays an important role for practicality purposes.

We consider a MIMO system with $N_{T}$ LED and $N_{R}$ PD (see Fig. 1). We use spatial multiplexing (SM) MIMO mode where different signals are transmitted from each LED. The achievable data rate $(R)$ with $M$-PAM can be calculated by

$$
R_{M-\mathrm{PAM}}=\frac{N_{T}}{T_{S}} \frac{N}{N+N_{C P}} \log _{2} M
$$

where $T_{S}$ is the sampling interval. Let $c_{n m}(t)$ and $h_{n m}(t)$ respectively denote the optical and electrical channel impulse response (CIR) from the $m^{t h}$ LED to the $n^{t h}$ PD. They are related to each other as, assuming electrical-to-optical conversion loss is unity,

$$
h_{n m}(t)=g_{t x}(t) \otimes c_{n m}(t) \otimes g_{r x}(t)
$$

It should be noted that for SM mode, $N_{R}$ should be equal to or greater than $N_{T}$. 
where $g_{t x}(t)$ and $g_{r x}(t)$ are transmit and matched-filter responses, respectively. The received signal on the $n^{\text {th }}$ PD can be written as

$$
y_{n}(t)=\sum_{m=1}^{N_{T}} x_{m}(t) \otimes h_{n m}(t)+v_{n}(t)
$$

where $x_{m}(t)$ is the transmitted signal from the $m^{t h}$ LED and $v_{n}(t)$ is additive white Gaussian noise (AWGN) term with zero mean and $\sigma_{N}^{2}$ variance at $n^{\text {th }}$ PD.

At the receiver side, for each received signal, $y_{n}(t)$, serialto-parallel conversion is performed, then $\mathrm{CP}$ is removed. This is followed by $N$-point DFT process such as

$$
Y_{n}[k]=\sum_{t=0}^{N-1} y_{n}[t] e^{-j \frac{2 \pi k t}{N}}
$$

The output of $n^{t h}$ path is denoted by $\mathbf{Y}_{n}$ with a dimension of $N$. For each $k$ where $k \in\{0,1 \ldots N-1\}$, a vector with the dimension of $N_{R}$ is defined such as $\mathbf{Y}_{\mathrm{eq}}^{k} \cdot n^{t h}$ element of $\mathbf{Y}_{\mathrm{eq}}^{k}$ is equal to $\mathbf{Y}_{n}[k]$ such as

$$
\mathbf{Y}_{\mathrm{eq}}^{k}=\left[\mathbf{Y}_{1}[k] \mathbf{Y}_{2}[k] \ldots \mathbf{Y}_{N_{R}}[k]\right]^{\mathrm{T}} .
$$

The zero-forcing (ZF) equalization process is further done through

$$
\overline{\mathbf{Y}}[k]=\mathbf{Y}_{\mathrm{eq}}^{k} \mathbf{W}_{n m}[k]
$$

where $\mathbf{W}_{n m}[k]$ includes $k^{t h}$ element of $N$-point DFT response of the channel between $m^{t h}$ LED and $n^{t h}$ PD whereas $\overline{\mathbf{Y}}$ is the equalized signal with the dimension of $N_{T}$. This process is repeated for each $k$, and the output is reconstructed with respect to each $m$ where $m \in\left\{1,2, \ldots N_{T}\right\}$. Equalized signal transmitted from $m^{\text {th }}$ LED is denoted by $\overline{\mathbf{Y}}_{m}$ with the dimension of $N$. Inverse discrete Fourier transform (IDFT) is performed over each $\overline{\mathbf{Y}}_{m}$ and the output is denoted by $\overline{\mathbf{y}}_{m}$. Transmitted symbols over $m^{\text {th }}$ LED and $k^{\text {th }}$ order within a frame are estimated through Maximum Likelihood (ML) decision rule which can be written as

$$
\hat{s}_{m}[k]=\underset{x \in \boldsymbol{\Phi}}{\operatorname{argmin}}\left\|\bar{y}_{m}[k]-x\right\|^{2}
$$

where $\boldsymbol{\Phi}$ denotes the symbols in deployed constellation scheme. Lower bound of BER for transmitted signal from $m^{t h}$ LED can be written as

$$
\mathrm{BER}_{M-\mathrm{PAM}}^{m} \geq \frac{2(M-1)}{M \log _{2} M} Q\left(\frac{1}{M-1} \sqrt{\frac{I^{2}}{\sigma_{N}^{2} \mathbf{E}\left[\left|\mathbf{W}_{m}^{\mathrm{T}}\right|^{2}\right]}}\right)
$$

and the overall BER can be found through taking the average of each BER calculated in (9) such as

$$
\mathrm{BER}=\frac{1}{N_{T}} \sum_{m=1}^{N_{T}} \mathrm{BER}_{M-\mathrm{PAM}}^{m} .
$$

\section{Channel Model}

We consider an office space with dimensions of $5 \mathrm{~m} \times$ $5 \mathrm{~m} \times 3 \mathrm{~m}$ (see Fig. 2) and 16 PDs (see Fig. 3) located at the ceiling. The source terminal is in the form of a laptop computer placed on the desk. It is connected to four USB hubs, where each USB hub is equipped with 4 LEDs (see Fig. 4). The separation between adjacent LEDs is taken as $5 \mathrm{~cm}$. The distance between adjacent USB hubs is set at $12.5 \mathrm{~cm}$. The coordinates of both LEDs and PDs are given in Table I in terms of $\mathrm{cm}$ where $(0,0,0)$ denotes the center of the floor. The other specifications are summarized in Table II.

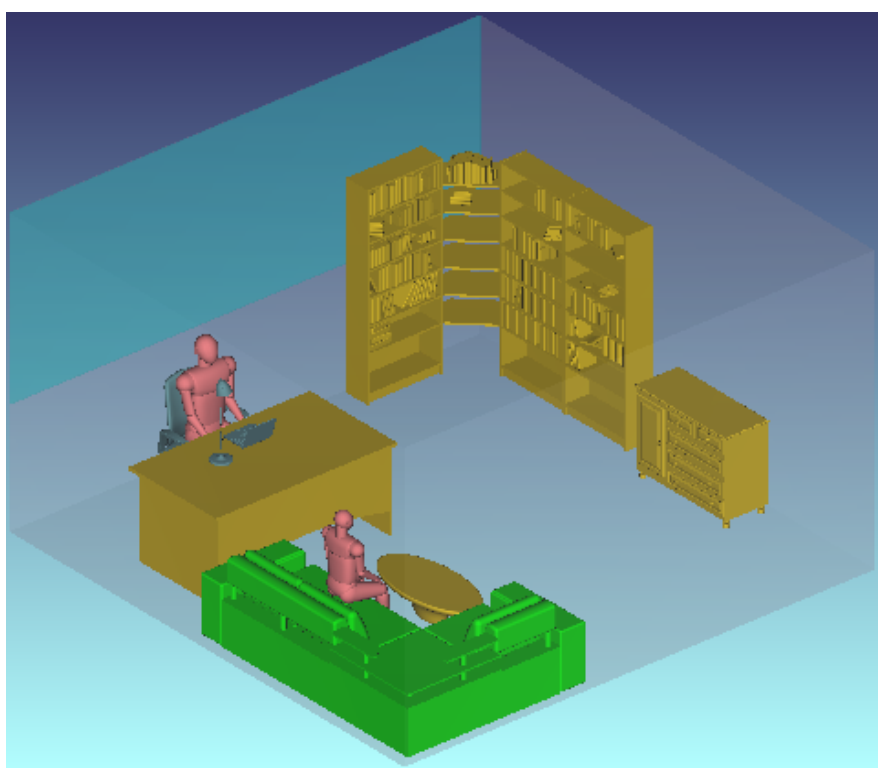

Figure 2: Top view of the office space.

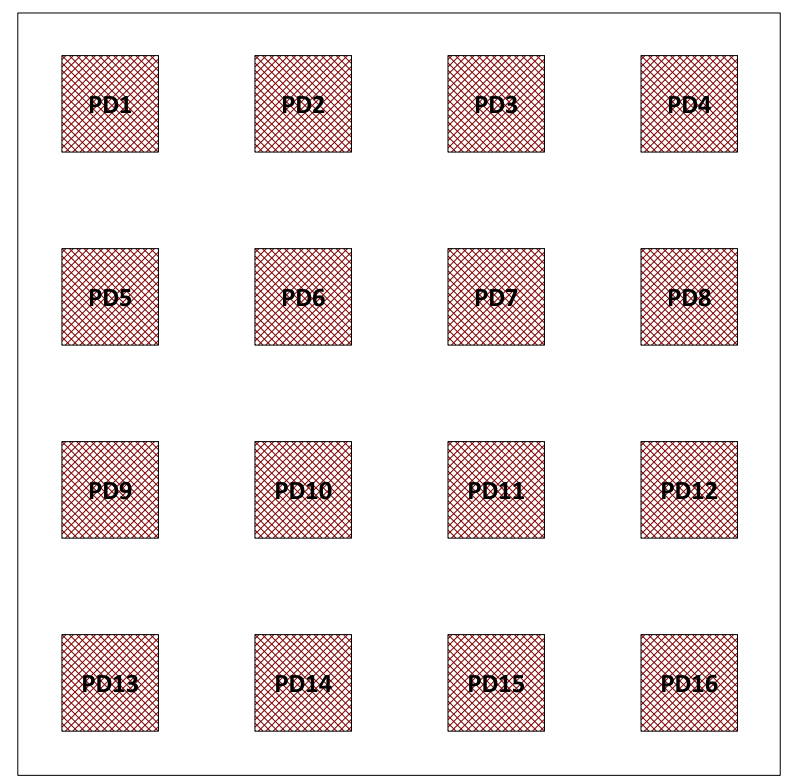

Figure 3: Arrangement of PDs on the ceiling (not to scale). 


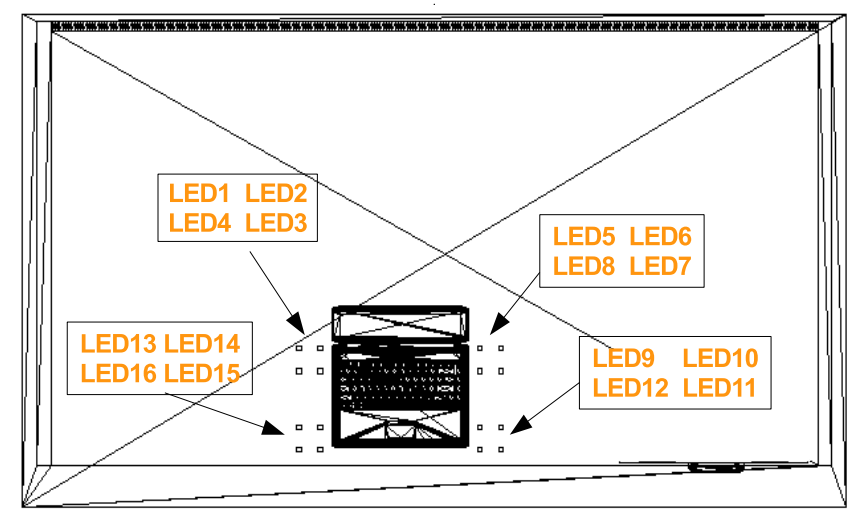

Figure 4: Top view of the desk with laptop computer and LEDs labeled from 1 to 16 .

Table I: Coordinates of LEDs and PDs

\begin{tabular}{|c|c|c|c|}
\hline Source & Coordinates & Destination & Coordinates \\
\hline LED1 & $(-777,1175,880)$ & PD1 & $(2090,2100,3000)$ \\
\hline LED2 & $(-827,1175,880)$ & PD2 & $(2090,700,3000)$ \\
\hline LED3 & $(-827,1225,880)$ & PD3 & $(2090,-700,3000)$ \\
\hline LED4 & $(-777,1225,880)$ & PD4 & $(2090,-2100,3000)$ \\
\hline LED5 & $(-1200,1175,880)$ & PD5 & $(690,2100,3000)$ \\
\hline LED6 & $(-1250,1175,880)$ & PD6 & $(690,700,3000)$ \\
\hline LED7 & $(-1250,1225,880)$ & PD7 & $(690,-700,3000)$ \\
\hline LED8 & $(-1200,1225,880)$ & PD8 & $(690,-2100,3000)$ \\
\hline LED9 & $(-1200,1350,880)$ & PD9 & $(-710,2100,3000)$ \\
\hline LED10 & $(-1250,1350,880)$ & PD10 & $(-710,700,3000)$ \\
\hline LED11 & $(-1250,1400,880)$ & PD11 & $(-710,-700,3000)$ \\
\hline LED12 & $(-1200,1400,880)$ & PD12 & $(-710,-2100,3000)$ \\
\hline LED13 & $(-777,1350,880)$ & PD13 & $(-2110,2100,3000)$ \\
\hline LED14 & $(-827,1350,880)$ & PD14 & $(-2110,700,3000)$ \\
\hline LED15 & $(-827,1400,880)$ & PD15 & $(-2110,-700,3000)$ \\
\hline LED16 & $(-777,1400,880)$ & PD16 & $(-2110,-2100,3000)$ \\
\hline
\end{tabular}

Let $h_{p, l}^{\mathrm{opt}}(t), l \in\{1,2 \ldots L\}, p \in\{1,2 \ldots P\}$ denote optical CIR for the link from the $l^{\text {th }}$ LED to the $p^{\text {th }}$ PD. Optical CIRs for each link are obtained using ray tracing simulations similar to those in [17]. As an example, we present CIRs for the first PD, i.e., $h_{1, l}^{\mathrm{opt}}(t), l \in\{1,2, \ldots 16\}$ in Fig. 5. In addition to the multipath propagation environment, the lowpass filter nature of IR LEDs should be further taken into account as depicted in Fig. 6. The frequency response of LEDs is commonly modelled as [18]

$$
H_{\mathrm{LED}}(f)=\frac{1}{1+j \frac{f}{f_{\text {cut }- \text { off }}}},
$$

where $f_{\text {cut-off }}$ is the LED $3-\mathrm{dB}$ cut-off frequency (see Fig. 6 in which $H_{\mathrm{LED}}(f)$ is depicted for $\left.f_{\text {cut-off }}=2 \mathrm{MHz}\right)$. The end-to-end channel frequency response taking into account the LED characteristics can be then expressed as $H_{p, l}^{\mathrm{E} 2 \mathrm{E}}(f)=$ $H_{\mathrm{LED}}(f) H_{p, l}^{\mathrm{opt}}(f)$ where $H_{p, l}^{\mathrm{opt}}(f)=F\left\{h_{p, l}^{\mathrm{opt}}(t)\right\}$.

\section{NUMERICAL RESULTS}

We investigate the BER and PAPR performance of SCFDE based MIMO uplink transmission over realistic infrared channels with respect to different modulation orders. During simulations, square root raised cosine (SRRC) with roll-off
Table II: Office room model specifications.

\begin{tabular}{|l|l|}
\hline \multirow{3}{*}{ Materials } & Walls: Plaster, \\
& Ceiling: Plaster, \\
& Floor: Pinewood, \\
& Desk: Pinewood \\
\hline \multirow{5}{*}{ Objects } & 1 desk and a chair paired with desk, \\
& 1 laptop on the desk, \\
& 1 desk light on the desk, \\
& 1 library, 1 couch, 1 coffee table, \\
& window, 2 human bodies \\
\hline \multirow{5}{*}{ Object specifications } & Desk: Pinewood (Height 0.88 m), \\
& Chair: Black gloss paint, \\
& Laptop: Black gloss paint \\
& Desk light: Black gloss paint, \\
& Library: Pinewood, \\
& Window: Glass \\
& Couch: Cotton, \\
& Coffee table: Pinewood \\
& Human body: Head \& Hands \\
& (assumed to be absorbing), \\
& clothes (cotton), shoes (black gloss) \\
\hline
\end{tabular}

factor of 0.5 is considered as transmit and receive pulse shaping filters. LED 3-dB cut-off frequency, sampling interval, noise power spectral density (PSD), frame size, cyclic prefix length are set to $2 \mathrm{MHz}, 250 \mathrm{nsec}, 10^{-22} \mathrm{~W} / \mathrm{Hz}, 64$ and 16 .

\section{A. BER Performance}

In Fig. 7, we show the BER performance results of SC-FDE based uplink transmission with respect to unipolar 2-PAM, 4-PAM, 8-PAM, and 16-PAM. The lower bounds obtained from (10) are also included at the same figure and depicted with ' $\mathrm{O}$ '. The results indicate that $16.9 \mathrm{dBm}$ mean emitted optical power is required to achieve the data rate of $51.2 \mathrm{Mbps}$ with the target BER of $10^{-3}$ using 2-PAM. Furthermore, 4-PAM which approximately doubles the data rate requires $21.3 \mathrm{dBm}$ optical power to achieve the same BER target. The amount of optical power should be further increased to 24.9 $\mathrm{dBm}$ and $28.1 \mathrm{dBm}$ for 8-PAM and 16-PAM which provide 153.6 Mbps and 204.8 Gbps data rates, respectively, with $10^{-3}$ BER target.

\section{B. PAPR Performance}

LEDs have limited linear region and amplifiers that can be utilized may cause signal distortion and clipping due to high PAPR. Moreover, high PAPR increases complexity of analogto-digital and digital-to-analog converters due to an increase in quantization. Hence, low PAPR of the modulation scheme is favorable for practicality purposes.

In Fig. 8 we provide the PAPR performance comparison for different unipolar PAM orders. The results show that 2-PAM has the lowest PAPR. The probability of PAPR greater than a threshold of $3.3 \mathrm{~dB}$ is 0.05 . Same threshold value becomes $4.39 \mathrm{~dB}$ for $4-\mathrm{PAM}$ at the same probability of 0.05 . The value is further increased to $4.75 \mathrm{~dB}$ and $4.9 \mathrm{~dB}$ for $8-\mathrm{PAM}$ and 16-PAM. IR LEDs have limited linear working region and the peak power point of the linear region should not be exceeded. In other words, PAPR value added to the required power level for target BER should be lower than the peak power point 

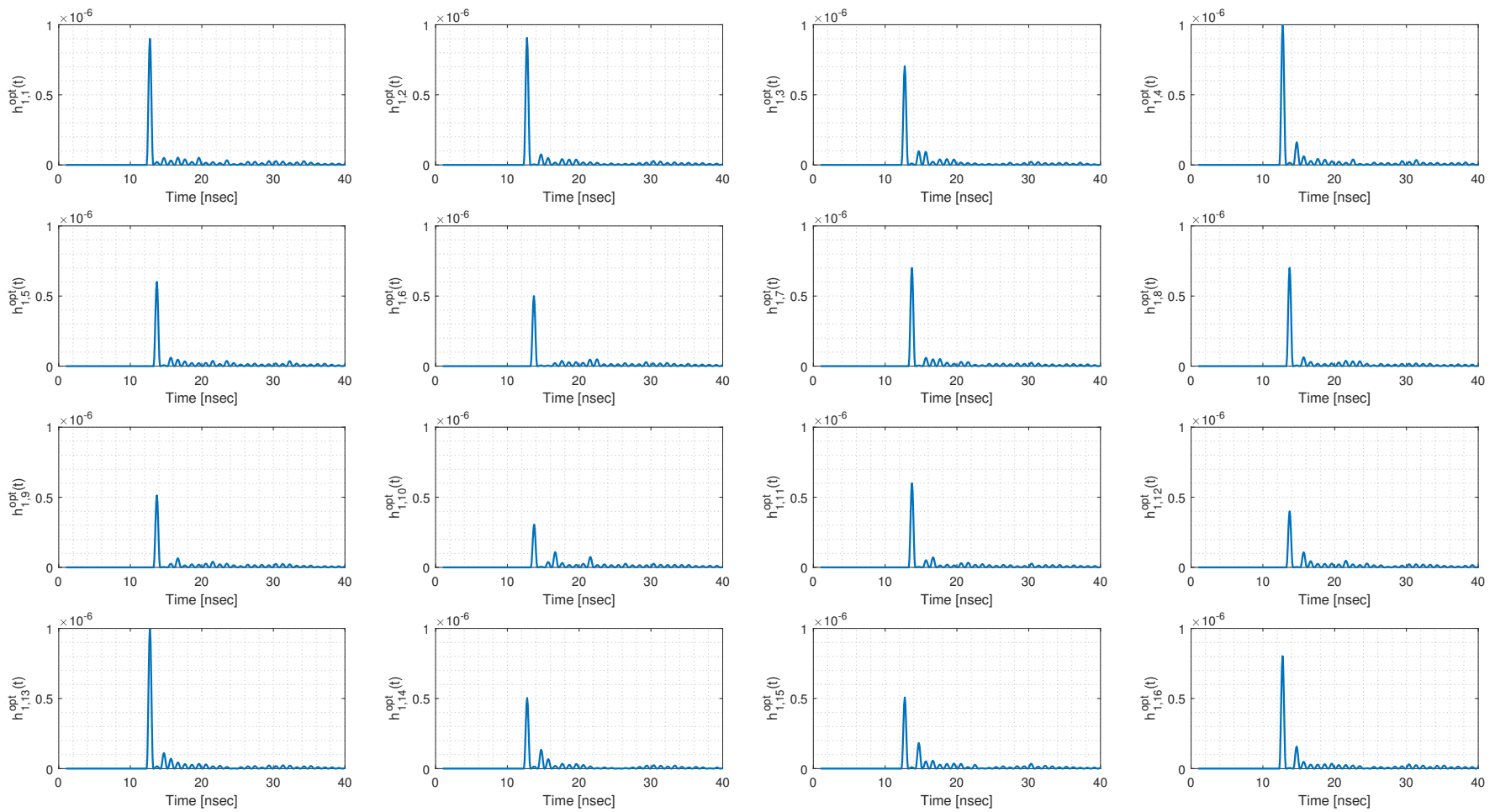

Figure 5: Optical CIRs for the first PD.

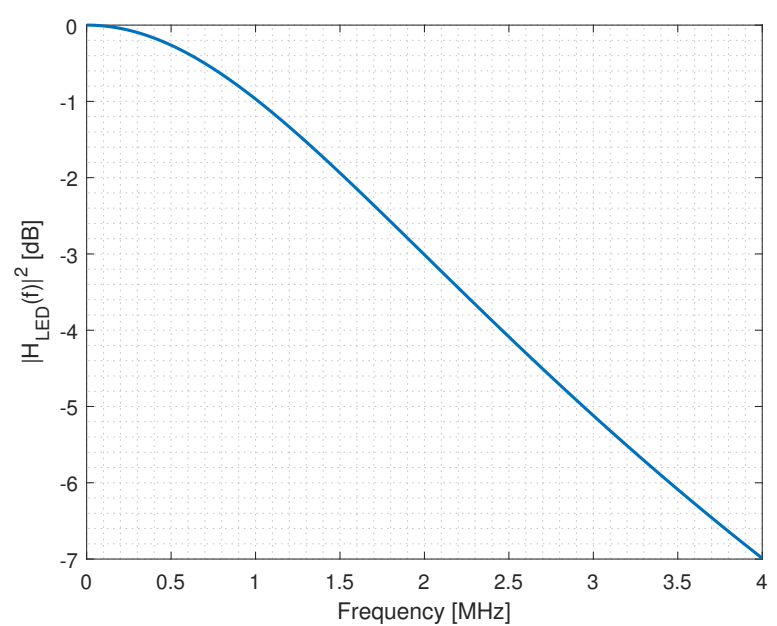

Figure 6: LED frequency response for $f_{\text {cut-off }}=2 \mathrm{MHz}$.

of the linear region to practically utilize the IR LED under consideration.

\section{CONCLUSION}

In this paper, we have proposed an SC-FDE based uplink transmission scheme for IR LED based optical communications. The proposed scheme utilizes $16 \times 16$ multiplexing MIMO mode. BER and PAPR performance of the proposed transmission is evaluated over realistic IR channels that are obtained through ray-tracing simulations including IR LED

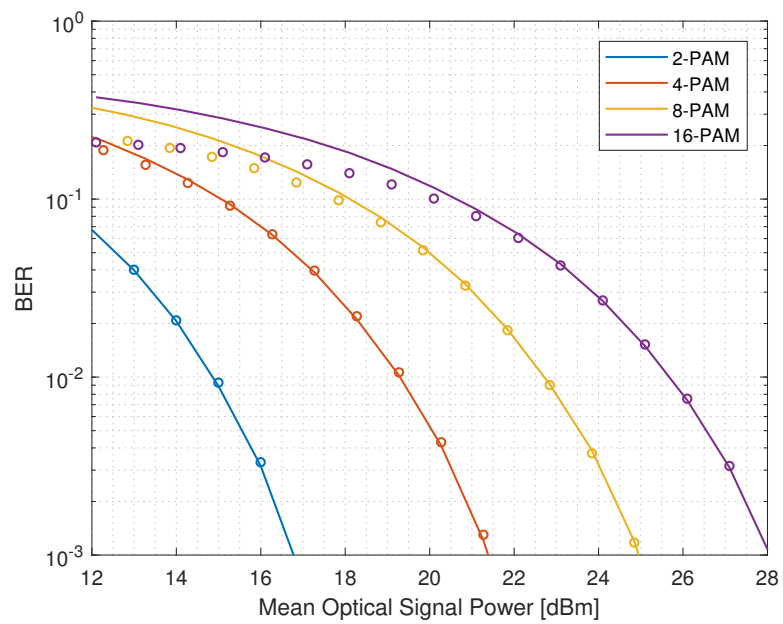

Figure 7: BER performance of SC-FDE MIMO transmission with respect to different modulation orders.

low-pass filter characteristics. It can be concluded that, SCFDE offering higher data rates with its lower PAPR advantage compared to multi-carrier schemes, is a promising technique to be used with IR LEDs for uplink transmissions.

\section{REFERENCES}

[1] J. Armstrong, "OFDM for optical communications," Journal of Lightwave Technology, vol. 27, no. 3, pp. 189-204, 2009.

[2] J. Armstrong and A. J. Lowery, "Power efficient optical OFDM," Electronics Letters, vol. 42, pp. 370-372, March 2006. 


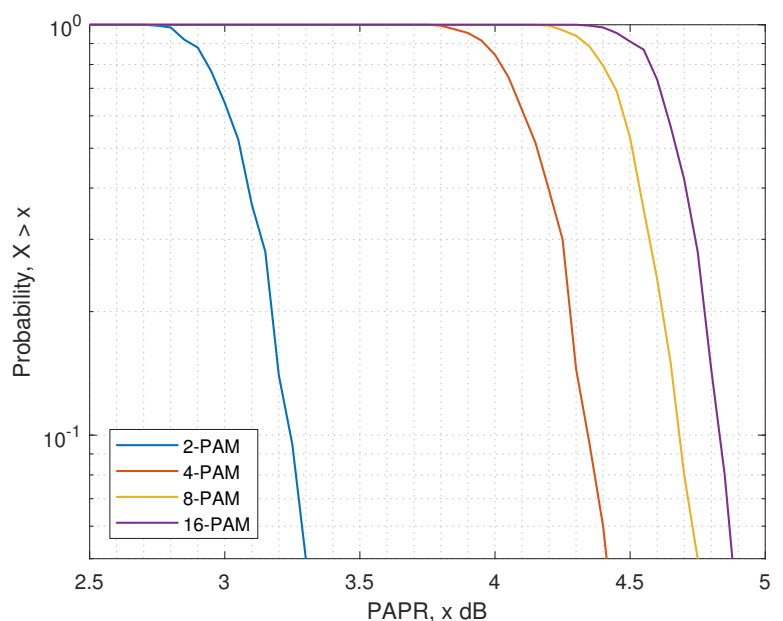

Figure 8: PAPR of SC-FDE MIMO transmission with respect to different modulation orders.

[3] N. Fernando, Y. Hong, and E. Viterbo, "Flip-OFDM for Unipolar Communication Systems," IEEE Transactions on Communications, vol. 60 , pp. 3726-3733, December 2012.

[4] D. Tsonev, S. Sinanovic, and H. Haas, "Novel Unipolar Orthogonal Frequency Division Multiplexing (U-OFDM) for Optical Wireless," in 2012 IEEE 75th Vehicular Technology Conference (VTC Spring), pp. 15, May 2012.

[5] D. Tsonev, S. Videv, and H. Haas, "Unlocking Spectral Efficiency in Intensity Modulation and Direct Detection Systems," IEEE Journal on Selected Areas in Communications, vol. 33, pp. 1758-1770, Sept 2015.

[6] M. A. Khalighi, S. Long, S. Bourennane, and Z. Ghassemlooy, "Pamand cap-based transmission schemes for visible-light communications," IEEE Access, vol. 5, pp. 27002-27013, 2017.

[7] Y. R. Zheng and C. Xiao, "Channel estimation for frequency-domain equalization of single-carrier broadband wireless communications," IEEE Transactions on Vehicular Technology, vol. 58, no. 2, pp. 815-823, 2009.

[8] M. Wolf, L. Grobe, M. R. Rieche, A. Koher, and J. Vuĉić, "Block transmission with linear frequency domain equalization for dispersive optical channels with direct detection," in Transparent Optical Networks (ICTON), 2010 12th International Conference on, pp. 1-8, IEEE, 2010.

[9] K. Acolatse, Y. Bar-Ness, and S. K. Wilson, "Novel techniques of singlecarrier frequency-domain equalization for optical wireless communications," EURASIP Journal on Advances in Signal Processing, vol. 2011, p. 4, 2011.

[10] F. Pancaldi, G. M. Vitetta, R. Kalbasi, N. Al-Dhahir, M. Uysal, and H. Mheidat, "Single-carrier frequency domain equalization," IEEE Signal Processing Magazine, vol. 25, no. 5, 2008.

[11] A. Yesilkaya, E. Basar, F. Miramirkhani, E. Panayirci, M. Uysal, and H. Haas, "Optical mimo-ofdm with generalized led index modulation," IEEE Transactions on Communications, 2017.

[12] L. Wu, Z. Zhang, and H. Liu, "Mimo-ofdm visible light communications system with low complexity," in Communications (ICC), 2013 IEEE International Conference on, pp. 3933-3937, IEEE, 2013.

[13] A. H. Azhar, T. Tran, and D. O'Brien, "A gigabit/s indoor wireless transmission using mimo-ofdm visible-light communications," IEEE photonics technology letters, vol. 25, no. 2, pp. 171-174, 2013.

[14] Y. Wang and N. Chi, "Indoor gigabit 2 ë 2 imaging multiple-inputmultiple-output visible light communication," Chinese Optics Letters, vol. 12, no. 10, p. 100603, 2014.

[15] M. T. Alresheedi, A. T. Hussein, and J. M. Elmirghani, "Uplink design in vlc systems with ir sources and beam steering," IET Communications, vol. 11, no. 3, pp. 311-317, 2017.

[16] T. Fath and H. Haas, "Performance Comparison of MIMO Techniques for Optical Wireless Communications in Indoor Environments," IEEE Transactions on Communications, vol. 61, pp. 733-742, February 2013.
[17] F. Miramirkhani and M. Uysal, "Channel Modeling and Characterization for Visible Light Communications," IEEE Photonics Journal, vol. 7, pp. 1-16, Dec 2015.

[18] L. Grobe and K. D. Langer, "Block-based PAM with frequency domain equalization in visible light communications," in 2013 IEEE Globecom Workshops (GC Wkshps), pp. 1070-1075, Dec 2013. 